\title{
Rhabdomyolysis following renal autotransplantation
}

\author{
Bryce Weber, MD; ${ }^{*}$ Gerald Todd MD; ${ }^{\dagger}$ Ronald B. Moore, MD, PhD ${ }^{\dagger}$
}

\begin{abstract}
A 26-year-old male body builder diagnosed with renal artery stenosis and middle aortic syndrome underwent an autotransplantation with bench reconstruction and end-to-end anastomosis using the hypogastric artery. Shortly after the procedure, the patient developed rhabdomyolysis and renal insufficiency, possibly related to his increased muscle mass, potentially greater susceptibility to hypertrophic skeletal muscle cells or his unique vascular condition. We review the risk factors, diagnosis, management and outcome of a case of rhabdomyolysis in a male patient who underwent autotransplantation for renal vascular hypertension.
\end{abstract}

CUAJ 2008;2(1):57-60

\section{Introduction}

Rhabdomyolysis is the dissolution or disintegration of muscle, associated with membrane lysis and leakage of muscle constituents, resulting in the excretion of myoglobin in the urine. It is a rare postoperative complication. ${ }^{1}$ Severe myalgia, unexplained muscle weakness and elevated creatine phosphokinase (CPK) are key to the diagnosis. Elevation of serum CPK, although diagnostic of myolysis, has poor correlation with the severity of subsequent renal failure. ${ }^{2}$ A rapid drop in serum myoglobin levels after the onset of oliguric renal failure suggests nonrenal clearance of myoglobin. ${ }^{2}$ The accompanying extracellular fluid volume contraction appears to be the principal determinant of the renal toxicity of myoglobinemia.

The clinical picture is one of oligoanuria, rapidly rising creatinine and hyperkalemia caused by potassium release from muscle cells. The diagnosis of rhabdomyolysis, however, is not straightforward, and a high index of suspicion is needed to make the diagnosis. Hemoglobinuric acute renal failure is usually caused by massive hemolysis, which may be seen during exposure to extracorporeal circulation or cardiopulmonary bypass. ${ }^{3}$ Major transfusion reactions that trigger anaphylactic shock, septicemia or transurethral resection of the prostate syndrome may lead to renal injury. ${ }^{2}$ Gluteal claudication has been reported with the use of the hypogastric artery for renal transplantation. ${ }^{4}$ In this case report, we review the risk factors, diagnosis, management and outcome of a case of rhabdomyolysis in a male patient undergoing autotransplantation for renal vascular hypertension.

\section{Case report}

A 26-year-old 125-kg male body builder was referred by his internal medicine specialist for management of refractory renal vascular hypertension. His hypertension was poorly controlled on 3 antihypertensives (metoprolol, 50 mg twice daily; coversyl, 4 mg daily; and hydrochlorothiazide, $25 \mathrm{mg}$ daily). Prior to his referral, the patient was diagnosed with middle aortic syndrome unsuitable for angioplasty. A magnetic resonance angiogram of the aorta revealed a narrowed aorta, a markedly stenotic celiac artery and an occluded superior mesenteric artery with a mesenteric circulation based on an enlarged inferior mesenteric and collateral marginal artery. Associated with this, there were 3 markedly stenotic renal arteries originating from the aorta on the right, with the 2 largest having ostium within $2 \mathrm{~mm}$ of each other. Two renal arteries were identified on the left, with no evidence of stenosis. The patient had no systemic symptoms of vasculitis, atherosclerotic disease, hypercoagulability or any gestalt of a connective tissue disorder. Consequently, the cause of his vascular abnormalities was thought to be congenital owing to the degree of collateralized vascular development (Fig. 1).

The patient was admitted to the hospital the same day of surgery through the pre-admission clinic. He was taken to the operating room where he underwent difficult placement of an epidural catheter followed by administration of general anesthesia. Following an uncomplicated right donor nephrectomy in the left lateral decubitus position, the patient was then rolled into the supine position for autotransplantation of the right kidney to the hypogastric artery in the left iliac fossa. Bench reconstruction of the renal vasculature was carried 
out by a second team while the flank incision and graft site were being prepared. The autotransplant was carried out without complications. The duration of the total operation (including anesthetic) was 9 hours and 40 minutes.

There was no perioperative tachycardia $(<100$ beats/min), hypercapnia, acidemia and only a low level of postoperative hyperthermia $\left(37.9^{\circ} \mathrm{C}\right)$. However, shortly after the patient was taken to the postanesthesia care unit, he complained of gluteal pain and loss of motor power to his legs. There was concern about cord compression from a cord hematoma secondary to the epidural catheter, and an urgent CT scan was arranged.

On postoperative day 1 , the patient continued to complain of gluteal pain, dysthesia and loss of muscle power. There was a marked drop-off in urine output, and his serum creatinine rose precipitously to $349 \mu \mathrm{mol} / \mathrm{L}$ despite aggressive fluid resuscitation (confirmed by central venous pressure monitoring). Creatinine kinase was obtained and revealed a level of $237000 \mathrm{U} / \mathrm{L}$, and the patient subsequently underwent urgent dialysis. Renal scan demonstrated both kidneys were wellperfused with a picture of acute tubular necrosis (Fig. 2). The patient required hemodialysis support for 19 days before his kidneys recovered function. Postoperatively, the patient was seen in consultation by a neurologist, and an electromyogram assessment was carried out 2 weeks postinsult. The findings were consistent with postganglionic axon loss in the left tibial peroneal and tibial nerves, with evidence of bilateral lumbosacral reflexopathy secondary to patchy ischemia. A bone scan (Fig. 3)

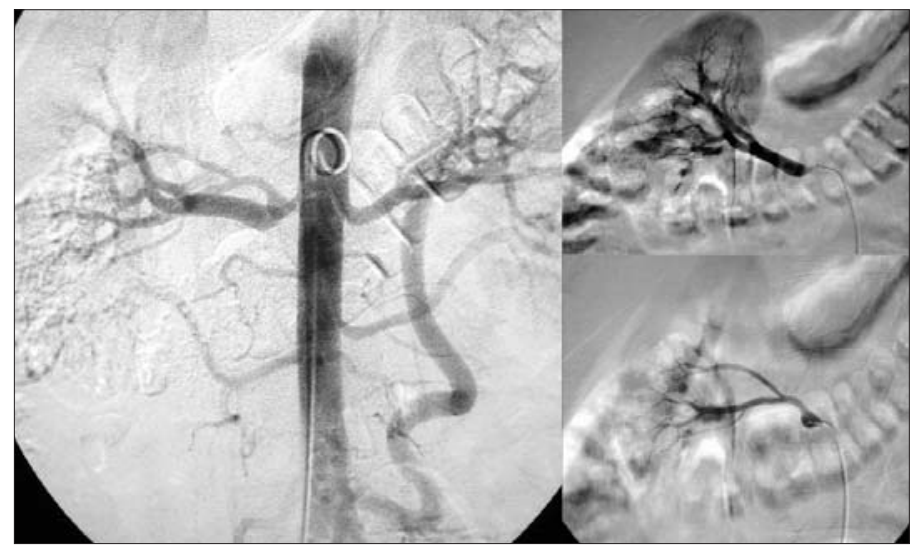

Fig. 1. Angiogram demonstrating middle aortic syndrome and markedly stenotic renal arteries originating from the aorta on the right, with the 2 largest arteries having ostium within $2 \mathrm{~mm}$ of each other (unsuitable for angioplasty). was carried out to differentiate between global and focal rhabdomyolysis, the former being consistent with malignant hyperthermia as opposed to pressure or hypoperfusion necrosis. The bone scan showed focal uptake in the gluteal region, particularly of the left. The patient was started on physiotherapy rehabilitation and was able to mobilize well with a walker and subsequently with canes. Following recovery of renal function and a follow-up renal scan demonstrating normal function of the left kidney and the autotransplanted right kidney, the patient was discharged home without further sequelae.

The patient continued to recover motor and sensory deficits over time, which posed only small limitations in function, several months from discharge. Additionally, his antihypertensive medications were gradually reduced until complete discontinuation 6 months after the operation. In the end, the patient was able to return to playing competitive hockey.

\section{Discussion}

Perioperative rhabdomyolysis may be caused by malignant hyperthermia, preoperative intensive muscular exercise, compression of musculature leading to muscle ischemia, dermatomyositis, one of several enzyme deficiencies and familial periodic paralysis. ${ }^{5-10}$ Muscular ischemia and malignant hyperthermia etiologies were deemed most likely in our patient because of the abrupt nature and timing of his rhabdomyolysis.

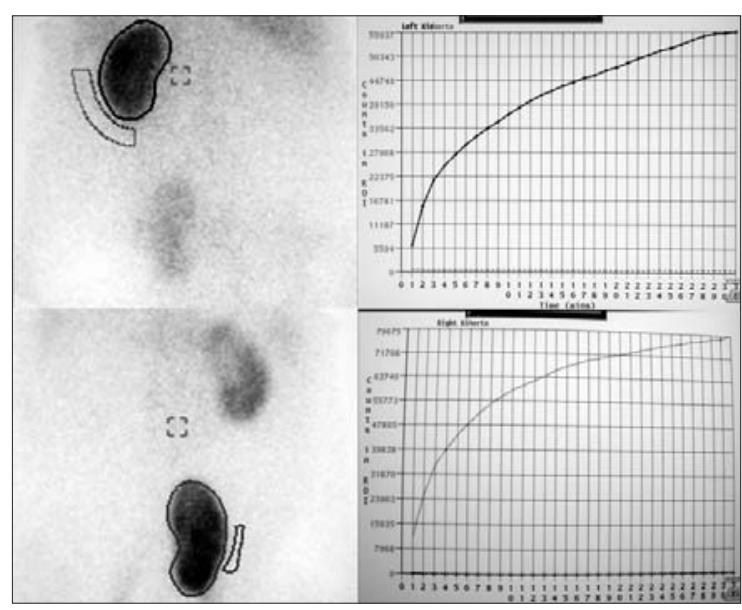

Fig. 2. Renal scan performed 24 hours after surgery showing both kidneys were well-perfused, with findings consistent with acute tubular necrosis. 
Many cases of surgical positioning-related rhabdomyolysis have been reported. ${ }^{5,8,11,12}$ Although this complication is most often reported for patients who undergo procedures while in a lithotomy position, it has also been reported for patients who undergo procedures while in a supine position, or a hyperlordodic posture, or for patients who simply undergo long-lasting surgery. ${ }^{6,12}$ Therefore, our patient's weight, the duration of his surgery and his positioning could potentially have lead to pressure ischemia. Conversely, a review of the literature did reveal that reported levels of creatinine kinase after intraoperative pressure-induced muscle ischemia were much lower $(<75000 \mathrm{U} / \mathrm{L})$ than that observed in our patient. ${ }^{\text {? }}$

Malignant hyperthermia was considered less likely because of the patient's lack of perioperative tachycardia, hypercapnia, acidemia and the low level of his postoperative hyperthermia. Because of the lack of reports that analyze case series of perioperative rhabdomyolysis, no judgment can be made on the validity of this phenomenon as an expression of malignant hyperthermia. However,

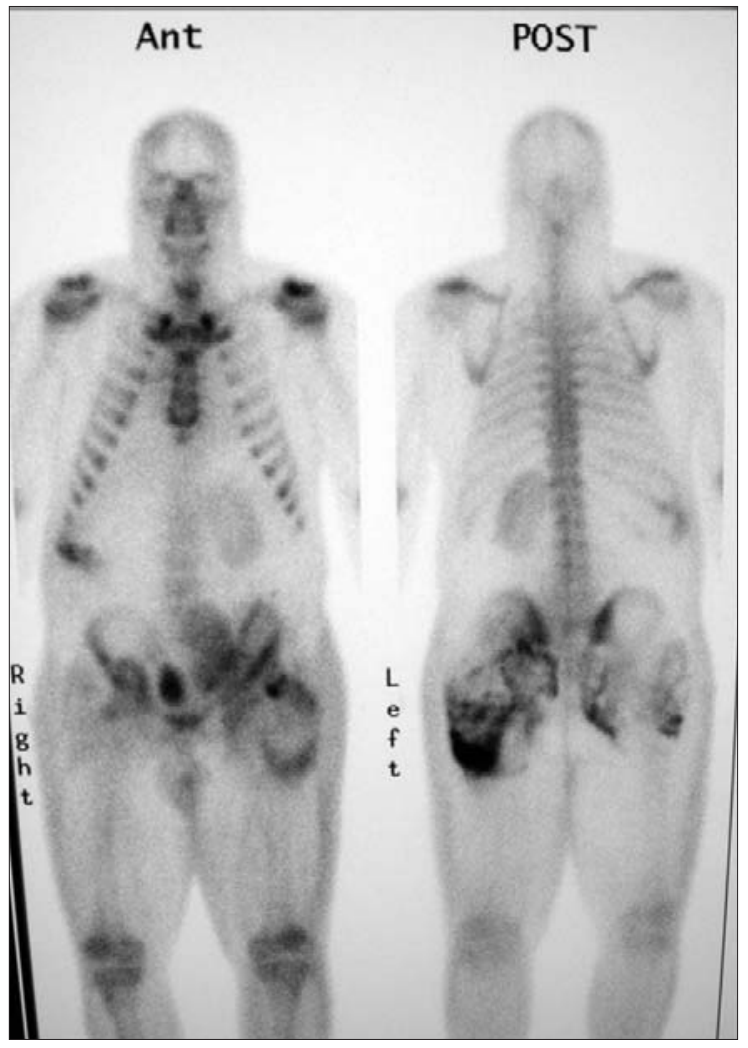

Fig. 3. A bone scan demonstrating focal uptake in the gluteal region, particularly on the left. in our case, the focal uptake on the bone scan would contradict this.

Creatinine kinase of skeletal muscle origin and its elevated level (237 $200 \mathrm{U} / \mathrm{L})$, along with the clinical findings, strongly suggest perioperative muscle cell destruction as the source of this patient's severe pain and myoglobinemia. These findings were consistent with length of operative time, the patient's massive gluteal muscles and the use of the hypogastric artery for manifold reconstruction of the renal artery. Therefore, it is reasonable to conclude that the gluteal muscles and lumbosacral plexus were injured by some mechanism (most likely vascular steal), and, as a result, a massive amount of myoglobin was released into the blood from the injured muscle cells. Increased muscle mass and the potentially greater susceptibility to hypertrophic skeletal muscle cells may have contributed to the presentation of rhabdomyolysis in this patient. Intense exercise or the possible intake of anabolic supplements could have influenced muscle metabolism and could have further aggravated the severity of the ischemic reaction. Also important to note was this patient's unique vascular condition, referred to as "middle aortic syndrome." As evident from the magnetic resonance angiogram images (Fig. 1), the middle abdominal aorta is quite abnormal with an irregular collateral blood supply. This vascular abnormality created a situation in which we felt it was best to avoid revascularization in situ with multiple jump grafts and elected bench reconstruction with autotransplantation to the hypogastric artery. The rationale behind this decision was to avoid the abnormal vasculature and to use the hypogastric manifold if required. The hypogastric artery end-to-end anastomosis, in this case, may have been involved in more collateral circulation than one would normally expect, leading to more extensive necrosis; however, this has not been described in the literature and was not evident in our workup.

In conclusion, we believe that our patient was at risk for rhabdomyolysis and renal insufficiency because of his increased muscle mass and at potentially greater susceptibility owing to hypertrophic skeletal muscle cells as well as his unique vascular condition, all of which created a questionable and unreliable situation. Thus a very high index of suspicion for rhabdomyolysis needs to be exercised when dealing with patients who have 
large muscle mass or unique vascular conditions and in whom the hypogastric artery is being used for renal revascularization.

From the *Division of Urology, Department of Surgery, University of Toronto, Toronto, Ont., and the †Division of Urology, Department of Surgery, University of Alberta, Edmonton, Alta.

This article has been peer reviewed.

Competing interests: None declared.

\section{References}

1. Dahlberg PJ, Howard RS. Rhabdomyolysis: an unusual postoperative complication. J Urol 1982;127:520-1.

2. Poels PJ, Gabreels FJ. Rhabdomyolysis: a review of the literature. Clin Neurol Neurosurg 1993;95:175-92.

3. Weldon BC, Monk TG. The patient at risk for acute renal failure. Recognition, prevention, and preoperative optimization. Anesthesiol Clin North America 2000;18:705-17.

4. Galazka Z, Szmidt J, Nazarewski S, et al. Kidney transplantation in recipients with atherosclerotic liliac vessels. Ann Transplant 1999;4:43-4.

5. Aschoff A, Steiner-Milz H, Steiner HH. Lower limb compartment syndrome following lumbar discectomy in the knee- chest position. Neurosurg Rev 1990;13:155-9.

6. Bertrand $M$, Godet $G$, Fleron MH, et al. Lumbar muscle rhabdomyolysis after abdominal aortic surgery. Anesth Analg 1997;85:11-5.

7. Harwood TN, Nelson TE. Massive postoperative rhabdomyolysis after uneventful surgery: a case report of subclinical malignant hyperthermia. Anesthesiology 1998;88:265-8.

8. Mathes DD, Assimos DG, Donofrio PD. Rhabdomyolysis and myonecrosis in a patient in the lateral decubitus position. Anesthesiology 1996;84:727-9.

9. Nimmo GR, Stewart SM, English PJ. Myoglobinuric acute renal failure associated with major urological surgery - An avoidable problem? Intensive Care Med 1998; 14:244-5.

10. Reneman RS. The anterior and the lateral compartmental syndrome of the leg due to intensive use of muscles. Clin Orthop Relat Res 1975;113:69-80.

11. Khalil IM. Bilateral compartmental syndrome after prolonged surgery in the lithotomy position. J Vasc Surg 1987;5:879-81.

12. Bukowski JG, Houet JF, Lepoitevin L, et al. Peroperative rhabdomyolysis caused by compression of a kidney-bridge. Value of muscular scintigraphy. Ann $\mathrm{Fr}$ Anesth Reanim 1989;8:659-61.

Correspondence: Dr. Bryce Weber, \#2, 191 Mutual St., Toronto ON M5B 2B4; bryce.weber@utoronto.ca

\section{Change of address}

We require 6 to 8 weeks' notice to ensure uninterrupted service. Please send your current mailing label, new address and the effective date of change to:

\section{CUA}

1155 University Ave., Suite 1155

Montréal QC H3B 3A7

journal@cua.org 